\title{
Case study for Performance Analysis of VOIP CODECS IN NON-MOBILITY SCENARIOS
}

\author{
Shreekant Gurrapu, Saurabh Mehta \& Shraddha panbude \\ Department of Electronics and Telecommunication, Vidyalankar Institute of Technology, \\ Mumbai India
}

\begin{abstract}
IEEE 802.11 is the most popular standard for WLAN networks. It offers different physical transmission rates. This paper focuses on this multi transmission rate of 802.11 WLANs and its effect on speech quality. In non-adaptive systems, when the physical layer switches from a higher transmission rate to a lower one, different than the one that the VoIP flow needs, the switching may result in congestion, high delay and packet loss, and consequently speech quality degradation. However, there are some algorithms that adapt the transmission parameters according to the channel conditions. In this study we demonstrate how choosing parameter (different codec and packet size) can affect the voice quality, network delay and packet loss. Further, this study presents a comparison between adaptive and non-adaptive methods. The adaptive method has also been evaluated for different congestion level from perceived speech quality point of view.
\end{abstract}

\section{KEYWORDS}

Adaptive rate control algorithm, Codec, IEEE 802.11, Multi-Rate WLAN, packetization interval, QoS, VoIP.

\section{INTRODUCTION}

There are a number of different techniques to handle the problem of multi-rate mechanism of 802.11 for speech traffic. On one hand, at the receiver side, playout buffer length (jitter buffer size) can be adaptive. This can be implemented to relieve delay and packet loss [1], [2], [3], [4] and [5]. On the other hand, rate adaptation mechanism can adjustthe input rate based on the link condition. One of the most famous rate adaptation techniques for speech transmission is codec switching or coding rate adjustment which can adjust different coding bit-rate (compression rate). Another one is by adjusting packetization interval. These techniques can be implemented in the sender side. In the transmission of VoIP (Voice over IP) traffic, rate adaptation mechanisms can be divided into two main categories; codec rate adaptation and adaptive packetization.

\subsection{VoIP}

Voice over IP (VoIP) is the real-time application that is probably the most widely-spread on today's networks. I'll provide here some basic facts related to VoIP. Figure below shows the endto-end path as needed for VoIP communication (a similar path exists in the opposite sense for a bi-directional connection). An audio input device, such as a microphone, is required at the sending end. The audio signal is transformed into digital form by an analog-to-digital converter. Due to the packet-switched nature of computer networks, voice data has to be packetized and encoded prior to being transmitted. Encoding (as well as decoding) is done by codecs that transform sampled voice data into a specific network-level representation and back. Most of the codecs are defined by standards of the International Telecommunication Union, the Telecommunication division (ITU-T). Each of them has different properties regarding the amount of bandwidth it requires but also the perceived quality of the encoded speech signal. After binary information is encoded and packetized at the sender end, packets encapsulating voice data can be transmitted on the network. Voice packets interact in the network with other application packets and are routed through shared connections to their destination. At the receiver end they 
are decapsulated and decoded. Decoding may include other steps as well, the most typical being dejittering. Other examples are error correction and packet loss concealment. The flow of digital data is then converted to analogue form again and played at an output device, usually a speaker.Wi-Fi is commonly used in residential, business, and public areas. It is notable that the perceived throughput in Wi-Fi does not match the real throughput. Also, all users share the access to the channel which is very critical for all real time traffic in general and especially VoIP. The low capacity in Wi-Fi connections has a high impact on the QoS in VoIP. Beside the high traffic generated by users, both protocols, VoIP and Wi-Fi, create large headers which result in high drawback on VoIP performance.

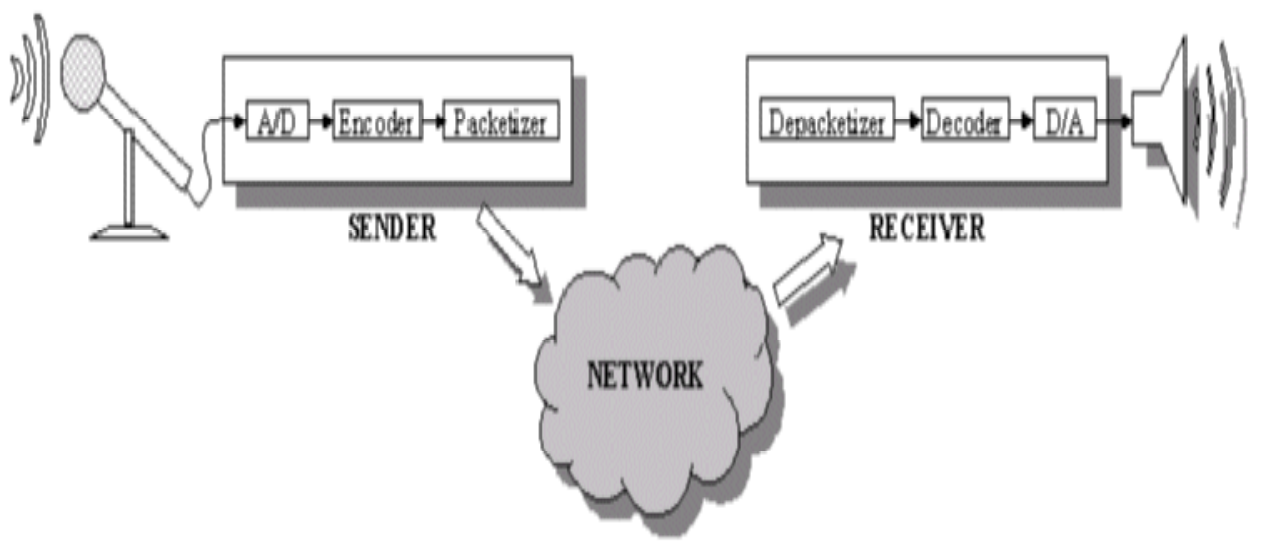

Fig.1 End-to-end data path for VoIP communication [1]

\subsection{QOS ISSUES OF VOIP APPLICATION}

Quality of Service (QoS) is what determines if a technology can successfully deliver high value services such as voice and video. [2] QoS is referred as the ability to control the mixture of bandwidth, delay, jitter, and packet loss in a network in order to deliver a network service [3] Ensuring high voice call quality over the "best effort" IP network is the key challenge in delivering VoIP traffic. QoS for VoIP is defined using different parameters, with the common ones being end-to-end delay, jitter and packet loss etc. The BE class which is used for data stream with no support for delay and throughput. In this paper, the quality of services for VoIP is measured in terms of end to end delay, jitter and packet loss. Delay or latency is defined as the time required for a frame or a packet to travel from the source to its final destination. The main source of delay is categorized into: Transmission Delay and destination processing delay, capacity calculation ineffective or insufficient, technological constraints, reordering packets queuing delay etc. [10] the variation of latency is jitter. An absolute value of delay difference between selected packets to arrive at destination is called as jitter. It is not guaranteed that all the packets will follow the same path and encounter the same routes to reach the destination over the network, and added with the congestion in the network usually resulting in packets arrival out of order and with varying delays. No jitter means a network with constant latency and no modification. The amount of data that can actually be transmitted over the communication channel is called throughput. It is used to Estimate the efficiency of network. The ratio between the quantity of information and the sum of use data, control data and retransmitted data if error is concluded as throughput of a network.

Nowadays, people get advantage of the existing data networks by enjoying various ways of communication e.g. text messages, voice calls, and video calls. The traditional phone networks cannot compete with these type of services due to low equipment's and operating cost, and the 
ability of integrating voice and data in applications. The QoS for VoIP can be measured by evaluating three performance metrics: Mean Opinion Score (MOS), Jitter, and end-to-end delay.

Mean Opinion Score (MOS): MOS is a scale from 1 to 5 which measures the quality of the voice. The value of worst quality is 1 and the best quality is 5 .

\begin{tabular}{|c|c|c|}
\hline Quality Scale & Score & Listening effort Scale \\
\hline Excellent & 5 & No effort required \\
\hline Good & 4 & No appreciable effort required \\
\hline Fair & 3 & Moderate effort required \\
\hline Poor & 2 & Considerable effort required \\
\hline Bad & 1 & No meaning understood with reasonable effort \\
\hline
\end{tabular}

Table 1. Mean Opinion Score (MOS) [1]

Jitter: The variation in arrival time of consecutive packets is called jitter. Beforedecoding, packets arrive to limited size buffer however some packets may lost orarrive out of order. Jitter can be calculated by computing the difference delayofpackets over a period of time.

Packet end-to-end delay: The end-to-end delay can be measured by calculating the delay from the speaker to the receiver. This includes network delay, encoding and decoding delay, and compression and decompression delay.

Table 1.2 shows the guidelines for voice quality measurement for both jitter and end-to- end delay as it is provided by ITU Telecommunication Standardization Sector (ITU-T).

\begin{tabular}{|l|c|c|c|}
\hline Network Parameter & Good & Acceptable & Poor \\
\hline Delay(ms) & $0-150$ & $150-300$ & $>300$ \\
\hline Jitter(ms) & $0-20$ & $20-50$ & $>50$ \\
\hline
\end{tabular}

Table 2. Guideline for the Voice Quality Measurement[1]

\subsection{CODEC AdAPTAtion}

Some codecs provide higher compression and as a result, lower utilization of bandwidth, so they support more calls. In the opposite side, some others provide lower compression resulting in higher bandwidth consumption and less number of calls. From another point of view, higher compression codecs has lower bit rate which means lower perceived quality. Two main speech codecs namely G.711 with $64 \mathrm{kbps}$ and G.729 with $8 \mathrm{kbps}$ bit rate widely used. G.729 utilizes one eight of the bandwidth compared to G.711. This means G.729 supports more calls but they are of lower quality. TheVoIP call that uses constant bit-rate codec has a fixed output rate, independent of network condition. Since a codec generates a constant output of bit rate, independent of network condition, lack of available bandwidth causes congestions and result in the packets being delayed or dropped.

Therefore, when the transmission rate is reduced, high bit rate codec can cause congestions while in same situation low bit rate codec can act normally. 


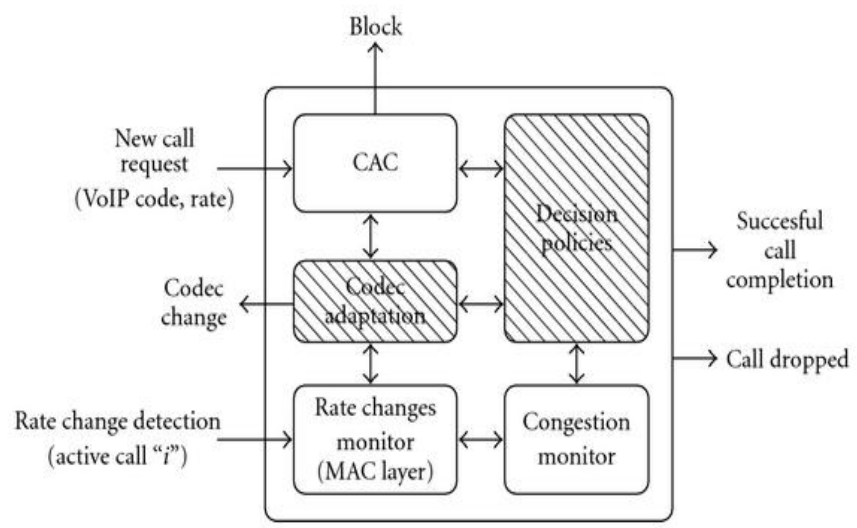

Fig.2. Codec adaptation [9]

\subsection{RATE ADAPTATION}

Rate adaptation is a link layer mechanism critical to the system performance by exploiting the multiple transmission rates provided by current IEEE 802.11 WLANs. The key challenge for designing such an algorithm is how to select the most appropriate transmission rate under different environments.

Rate adaptation is the determination of the optimal data transmission rate most appropriate for current wireless channel conditions. It consists of assessing channel conditions and accordingly adjusting the rate. Rate adaptation is fairly challenging due to wild channel conditions fluctuations. In the last decade, rate adaptation for IEEE 802.11 networks has been extensively investigated.

\subsection{NETWORK SIMULATOR 2}

Network Simulator NS-2 (Network Simulator) [8] is a discrete event driven simulator used for implementation and simulations of various network protocols.NS-2 was developed in the year 1989NS-2 is used for modelling network component like - Traffic models and applications: Web, FTP, telnet, audio, sensor nets - Transport protocols: TCP and UDP - Routing and Queuing, Local Area Networks, wireless links, and satellite links. NS-2 also provides infrastructure for tracing, visualization, error models and to modify or create our own modules. Using components in ns, different traffic and topologies can be generated and NAM (Network Animator) can be used for providing visual outputs. Advantages of NS-2 as a Simulation Tool NS-2 was chosen as the simulation tool for implementation of this paper because of its modular and open architecture.

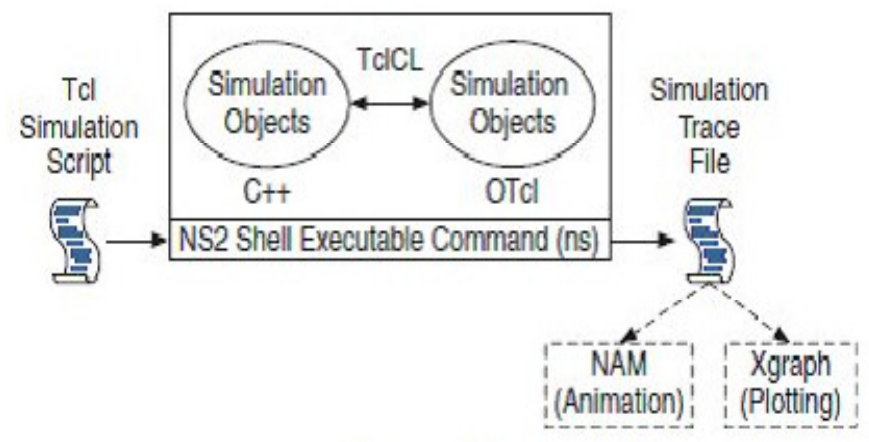

Fig 3. Basic Architecture of NS-2 Simulator [13] 
NS-2 supports dual output which can be either text-based or graphical based. For graphical based simulation NS-2 has inbuilt tool i.e. NAM (Network Animator). We have used the NS2 in our project due to easy to learning, user friendly, GUI as well as command line, open source, Linux based also real time and large number source capability.NS-2 is totally based on Object Oriented (OO)the simulator framework uses a two-language programming approach, OTcl and $\mathrm{C}++$. OTcl (object oriented version of $\mathrm{Tcl}$ ) is fast to run, whereas $\mathrm{C}++$ is fast to write and change. The framework allows a very detailed modeling of wireless communication using radio prorogation models, Antennas, link layer, ARP, MAC layer protocols (e.g. IEEE 802.11), as well as ad-hoc routing protocols like AODV, DSR, DSDV etc. Simulation Topology and Environment Following simulation parameters were adopted to test the validity of the modified approach $[8,10]$

\subsection{SimULATION SET UP}

Exponential traffic voice (created packet while talk period for $1.00 \mathrm{~ms}$ and no packet is created during $1.3 \mathrm{~ms}$ of silent period) is a scenario in the implementation using SIP to fulfill sending voice packet from end to end nodes.

\subsection{RESULT AND DISCUSSION}

In this Section, we compare the capabilities of the three VOIP codec's studied in this paper. To evaluate more reliable performance of G.711, G.723 and G.729 VoIP codec's in same simulation environment (50 to 300 mobile nodes). Performance metrics are calculated from trace file, with the help of AWK program. The simulation results shown in the following section in the form of line graph with description. The result shows the comparison between the three codecs on different QoS parameters in a VoIP network with Non-mobility scenarios.

\section{GRAPHS OF CODEC'S IN NON-MOBILITY SCENARIO - WI-FI:}

\subsection{CODEC ADAPTATION}

\subsubsection{Delay}

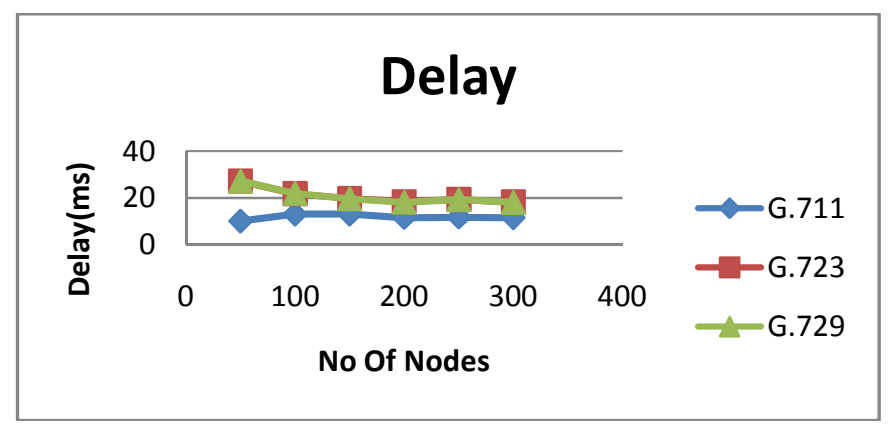

Fig.4. Delay vs Number of nodes

\begin{tabular}{|c|c|c|c|}
\hline \multicolumn{4}{|c|}{ Delay } \\
\hline No of Nodes & G.711 & $\mathbf{G . 7 2 3}$ & G.729 \\
\hline 50 & 10.006 & 27.1402 & 27.0985 \\
\hline 100 & 12.9204 & 21.8371 & 21.9207 \\
\hline 150 & 12.9789 & 19.7138 & 19.6157 \\
\hline 200 & 11.5245 & 18.402 & 18.0957 \\
\hline 250 & 11.7394 & 19.312 & 19.1733 \\
\hline 300 & 11.4341 & 18.1266 & 18.0227 \\
\hline
\end{tabular}

Table 3. Readings of Delay vs Number of nodes 
From the above figure and table Delay was gained at destination node against various dimension of networks and varied the simulation time uniformly for each codec. This data may be delivered over a physical or logical link, or pass through a certain network node. it is clear that G.729 gives more delay when the nodes are more. G.711 and G.723 codecs gives less delay. G.729 had a high delay. From these graphs it is clear that delay decrease with increase in non-mobility nodes.

\subsubsection{MOS}

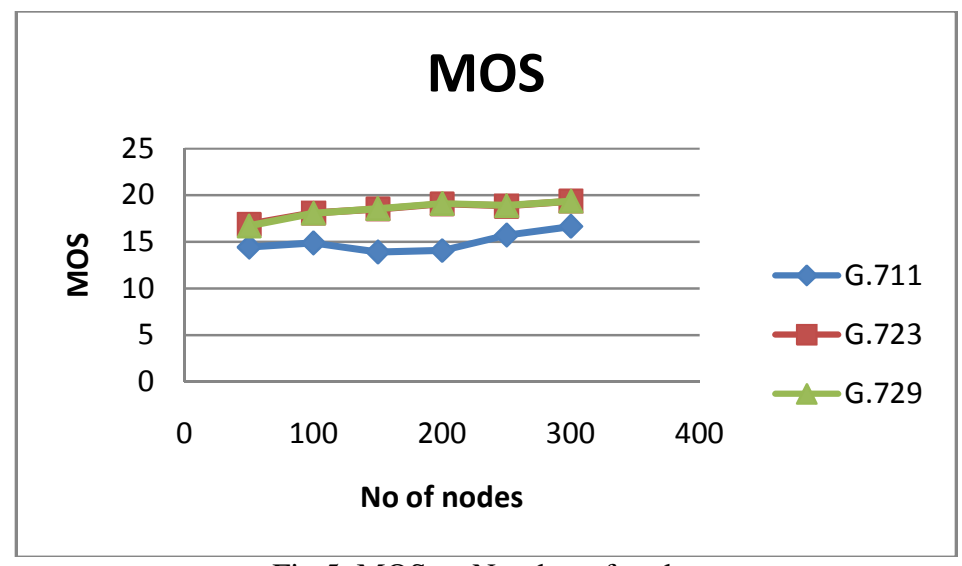

Fig.5. MOS vs Number of nodes

\begin{tabular}{|c|c|c|c|}
\hline \multicolumn{4}{|c|}{ MOS } \\
\hline No of Nodes & G.711 & G.723 & G.729 \\
\hline 50 & 14.4266 & 16.8844 & 16.7141 \\
\hline 100 & 14.8738 & 18.1194 & 18.0848 \\
\hline 150 & 13.8938 & 18.5201 & 18.5863 \\
\hline 200 & 14.0479 & 19.086 & 19.1325 \\
\hline 250 & 15.7118 & 18.8408 & 18.8946 \\
\hline 300 & 16.6547 & 19.3664 & 19.3738 \\
\hline
\end{tabular}

Table 4. Readings of MOS vs Number of nodes

The most widely used QoS metric in VOIP applications is MOS. The MOS value describes the voice perception quality. The average MOS value for the three codecs is represented in figure above. From the graph it is observed that MOS value increases as the nodes increases. Codecs G.711 and G.723 have acceptable MOS value G.723 and G.729, respectively. On the other hand, the MOS value for G.729 is 19.3738 which indicate that the quality of service is good if this codec is used.

\subsubsection{PDR}

Figure below figure and table describes the average voice PDR comparison using different codecs. From the figure, the variation of the codec is minimum after 100 nodes and approximately varying throughout the simulation. The average voice throughput variation in case of codec G.723 is lower than the other two codecs at the earlier time of simulation. The PDR variation in case of G.729 lies between two other audio codecs. So audio codec G.723 gives better results than audio codecs G.711 and G.729 respectively. 


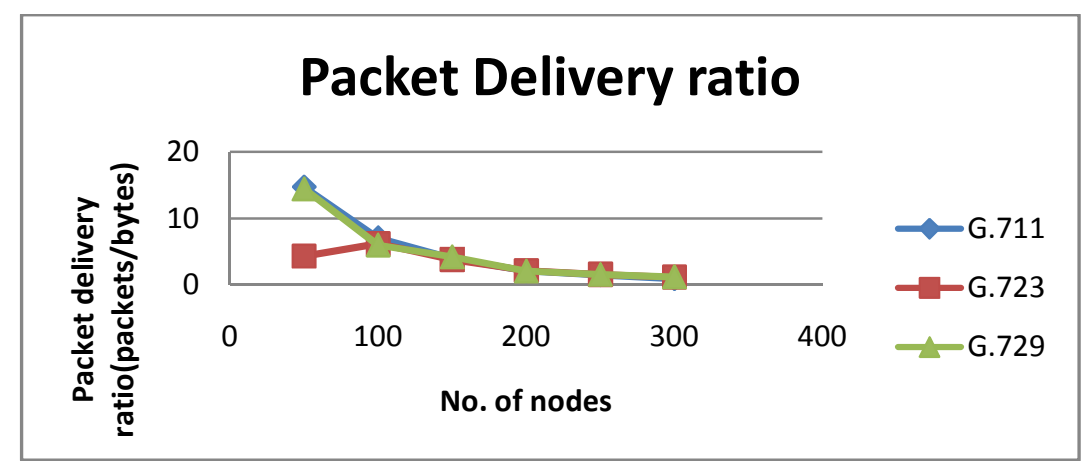

Fig.6. PDR vs Number of nodes

\begin{tabular}{|c|c|c|c|}
\hline & $\begin{array}{c}\text { Packet Delivery } \\
\text { ratio }\end{array}$ & & \\
\hline No of Nodes & G.711 & G.723 & G.729 \\
\hline 50 & 14.7053 & 4.2897 & 14.4356 \\
\hline 100 & 7.04911 & 6.16613 & 5.97557 \\
\hline 150 & 3.96553 & 3.79713 & 4.18666 \\
\hline 200 & 2.05143 & 2.14277 & 2.0679 \\
\hline 250 & 1.50003 & 1.5714 & 1.53505 \\
\hline 300 & 0.885652 & 1.14652 & 1.17401 \\
\hline
\end{tabular}

Table 5 Readings of PDR vs Number of nodes

\subsubsection{Throughput}

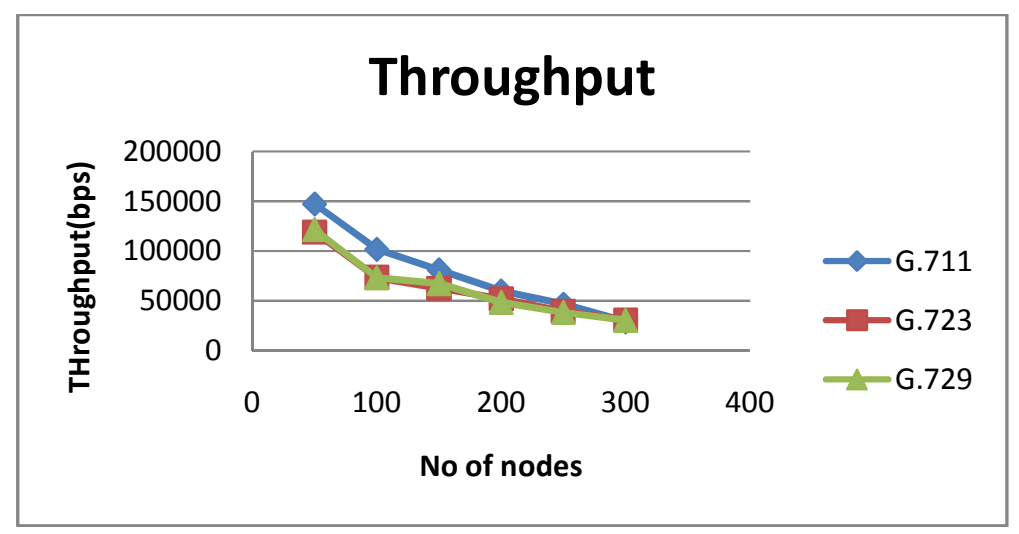

Fig.7. Throughput vs Number of nodes

\begin{tabular}{|c|c|c|c|}
\hline \multicolumn{5}{|c|}{ Throughput } \\
\hline No of Nodes & G.711 & G.723 & G.729 \\
\hline 50 & 146976 & 118617 & 121296 \\
\hline 100 & 101554 & 73229.2 & 73132.1 \\
\hline 150 & 80945.3 & 62387.5 & 67257.9 \\
\hline 200 & 59712.1 & 51735.5 & 48410.9 \\
\hline 250 & 46505.4 & 39101.3 & 38431.8 \\
\hline 300 & 28917.1 & 30617.7 & 30371.3 \\
\hline
\end{tabular}

Table 6. Readings of Throughput vs Number of nodes 
Figure above figure and table describes the average voice throughput comparison using different codecs. From the figure, the variation of the codec is minimum and approximately varying throughout the simulation. The average voice throughput variation in case of codec G.711 is higher than the other two codecs at the earlier time of simulation. But after some time it falls down. The throughput variation in case of G.723 lies between two other audio codecs. So audio codec G.729 gives better results than audio codecs G.711 and G.723 respectively.

\subsubsection{Jitter}

Wi-Fi is commonly used in residential, business, and public areas. It is notable that the perceived throughput in Wi-Fi does not match the real throughput. Also, all users share the access to the channel which is very critical for all real time traffic in general and especially VoIP. The low capacity in Wi-Fi connections has a high impact on the QoS in VoIP. Beside the high traffic generated by users, both protocols, VoIP and Wi-Fi, create large headers which result in high drawback on VoIP performance.

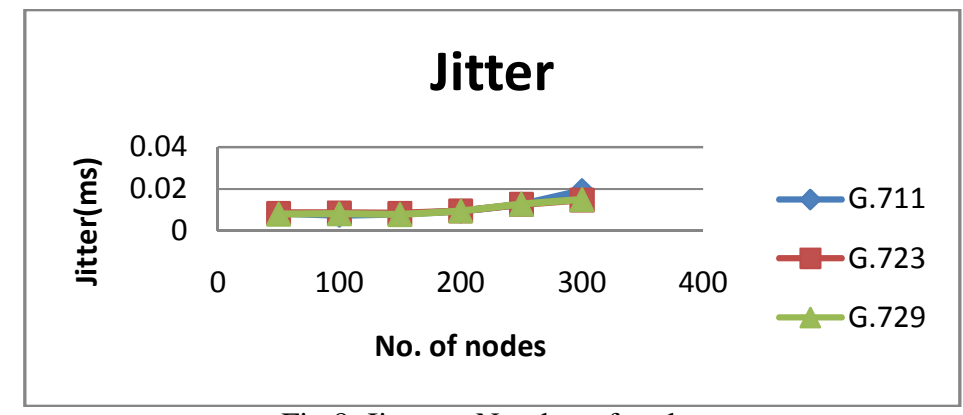

Fig.8. Jitter vs Number of nodes

\begin{tabular}{|c|c|c|c|}
\hline \multicolumn{4}{|c|}{ Jitter } \\
\hline No of Nodes & G.711 & G.723 & G.729 \\
\hline 50 & 0.00833 & 0.008498 & 0.008088 \\
\hline 100 & 0.007473 & 0.008641 & 0.008145 \\
\hline 150 & 0.008015 & 0.008401 & 0.00785 \\
\hline 200 & 0.009502 & 0.009492 & 0.009489 \\
\hline 250 & 0.012755 & 0.012794 & 0.012794 \\
\hline 300 & 0.019217 & 0.014816 & 0.014902 \\
\hline
\end{tabular}

Table 7. Readings of Jitter vs Number of nodes

\subsection{RATE AdAPTAtiaON}

\subsubsection{Delay}

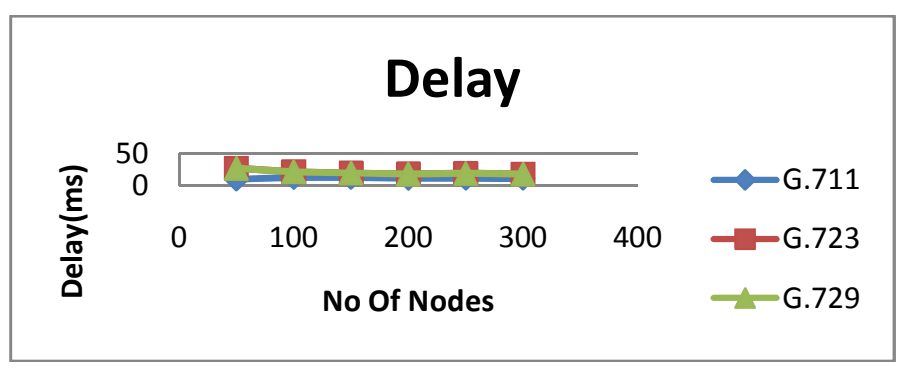

Fig. 9. Delay vs Number of nodes 


\begin{tabular}{|c|c|c|c|}
\hline \multicolumn{4}{|c|}{ Delay } \\
\hline No of Nodes & G.711 & G.723 & G.729 \\
\hline 50 & 10.006 & 27.1402 & 27.0985 \\
\hline 100 & 12.9204 & 21.8371 & 21.9207 \\
\hline 150 & 12.9789 & 19.7138 & 19.6157 \\
\hline 200 & 11.5245 & 18.402 & 18.0957 \\
\hline 250 & 11.7394 & 19.312 & 19.1733 \\
\hline 300 & 11.4341 & 18.1266 & 18.0227 \\
\hline
\end{tabular}

Table 8. Readings of Delay vs Number of nodes

From the above figure and table Delay was gained at destination node against various dimension of networks and varied the simulation time uniformly for each codec. This data may be delivered over a physical or logical link, or pass through a certain network node. It is clear that G.729 gives more delay when the nodes are more. G.711 and G.723 codecs gives less delay. G.729 had a high delay. From these graphs it is clear that delay decrease with increase in non-mobility nodes.

\subsubsection{MOS}

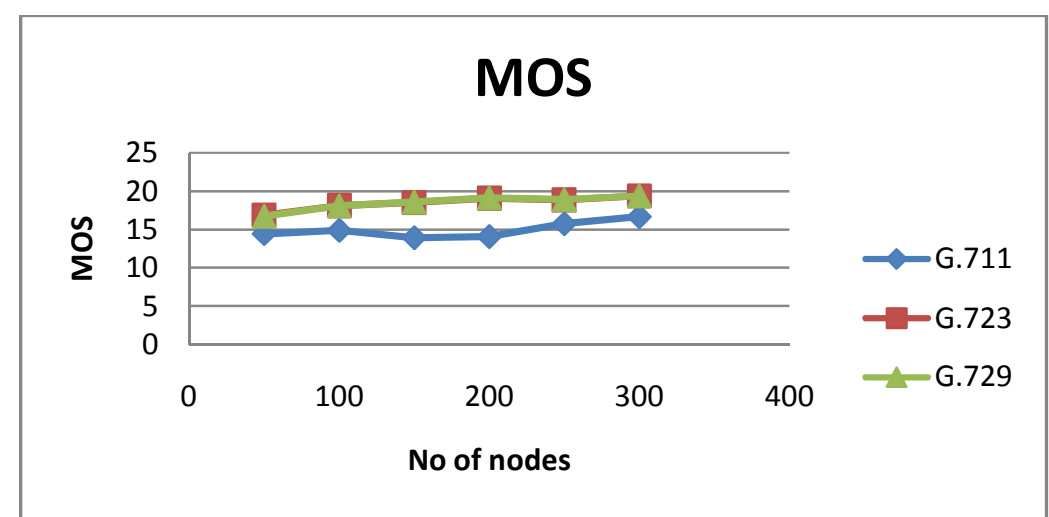

Fig.10. MOS vs Number of nodes

\begin{tabular}{|c|c|c|c|}
\hline \multicolumn{4}{|c|}{ MOS } \\
\hline No of Nodes & G.711 & $\mathbf{G . 7 2 3}$ & $\mathbf{G . 7 2 9}$ \\
\hline 50 & 14.4266 & 16.8844 & 16.7141 \\
\hline 100 & 14.8738 & 18.1194 & 18.0848 \\
\hline 150 & 13.8938 & 18.5201 & 18.5863 \\
\hline 200 & 14.0479 & 19.086 & 19.1325 \\
\hline 250 & 15.7118 & 18.8408 & 18.8946 \\
\hline 300 & 16.6547 & 19.3664 & 19.3738 \\
\hline
\end{tabular}

Table 9 Readings of MOS vs Number of nodes

The most widely used QoS metric in VOIP applications is MOS. The MOS value describes the voice perception quality. The average MOS value for the three codecs is represented in figure above. From the graph it is observed that MOS value increases as the nodes increases. Codecs G.711 and G.723 have acceptable MOS value G.723 and G.729, respectively. On the other hand, the MOS value for G.729 is 19.3738 which indicate that the quality of service is good if this codec is used. 


\subsubsection{PDR}

Figure below figure and table describes the average voice PDR comparison using different codecs. From the figure, the variation of the codec is minimum after 100 nodes and approximately varying throughout the simulation. The average voice throughput variation in case of codec G.723 is lower than the other two codecs at the earlier time of simulation.

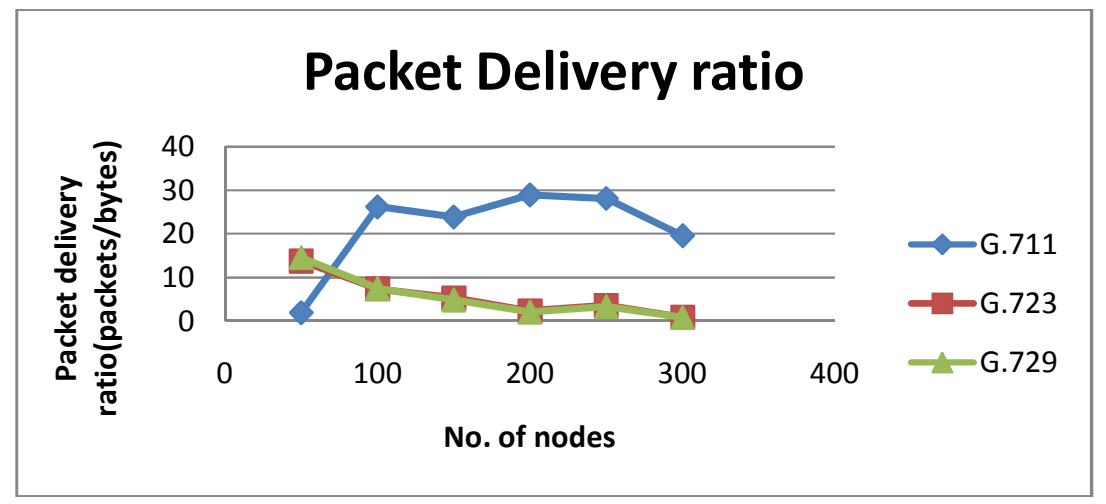

Fig.11 PDR vs Number of nodes

\begin{tabular}{|c|c|c|c|}
\hline \multicolumn{4}{|c|}{ Packet Delivery ratio } \\
\hline No of Nodes & G.711 & G.723 & G.729 \\
\hline 50 & 1.92308 & 13.7232 & 14.5958 \\
\hline 100 & 26.178 & 7.33656 & 7.51509 \\
\hline 150 & 23.8849 & 5.25982 & 4.91923 \\
\hline 200 & 28.8939 & 2.3436 & 2.10193 \\
\hline 250 & 28.1065 & 3.61075 & 3.33329 \\
\hline 300 & 19.5699 & 0.902837 & 0.86395 \\
\hline
\end{tabular}

Table 10. Readings of PDR vs Number of nodes

The PDR variation in case of G.729 lies between two other audio codecs. So audio codec G.723 gives better results than audio codecs G.711 and G.729 respectively.

\subsubsection{Throughput}

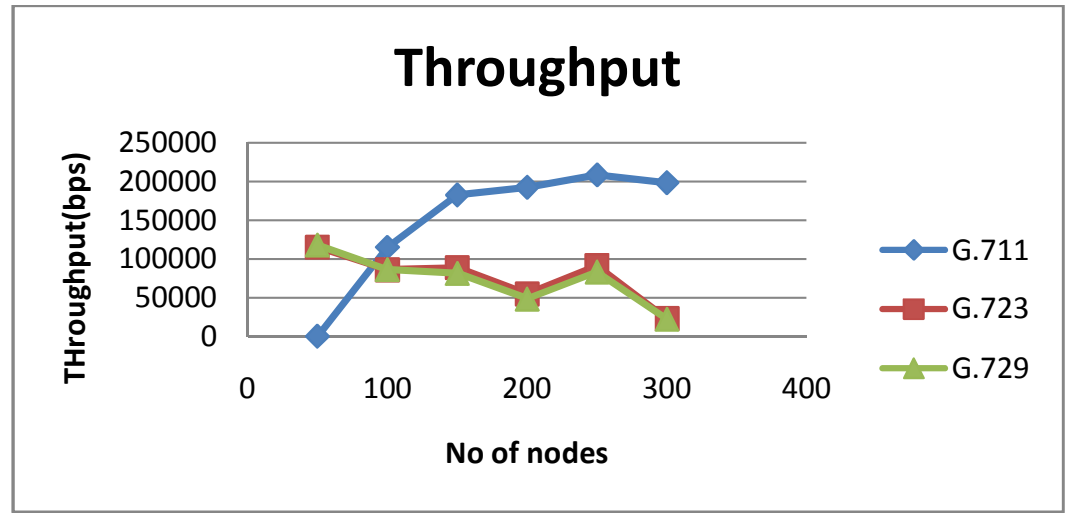

Fig. 12 Throughput vs Number of nodes 


\begin{tabular}{|c|c|c|c|}
\hline \multicolumn{4}{|c|}{ Throughput } \\
\hline No of Nodes & G.711 & G.723 & G.729 \\
\hline 50 & 738.091 & 115027 & 118334 \\
\hline 100 & 115282 & 86149.5 & 86526.2 \\
\hline 150 & 182626 & 88846.7 & 81689.5 \\
\hline 200 & 192470 & 55001 & 48734.8 \\
\hline 250 & 208345 & 91383.2 & 83598.3 \\
\hline 300 & 198229 & 23599.7 & 22636.6 \\
\hline
\end{tabular}

Table 11Readings of Throughput vs Number of nodes

Figure above figure and table describes the average voice throughput comparison using different codecs. From the figure, the variation of the codec is minimum and approximately varying throughout the simulation. The average voice throughput variation in case of codec G.711 is higher than the other two codecs at the earlier time of simulation. But after some time it falls down. The throughput variation in case of G.723 lies between two other audio codecs. So audio codec G.729 gives better results than audio codecs G.711 and G.723 respectively.

\subsubsection{Jitter}

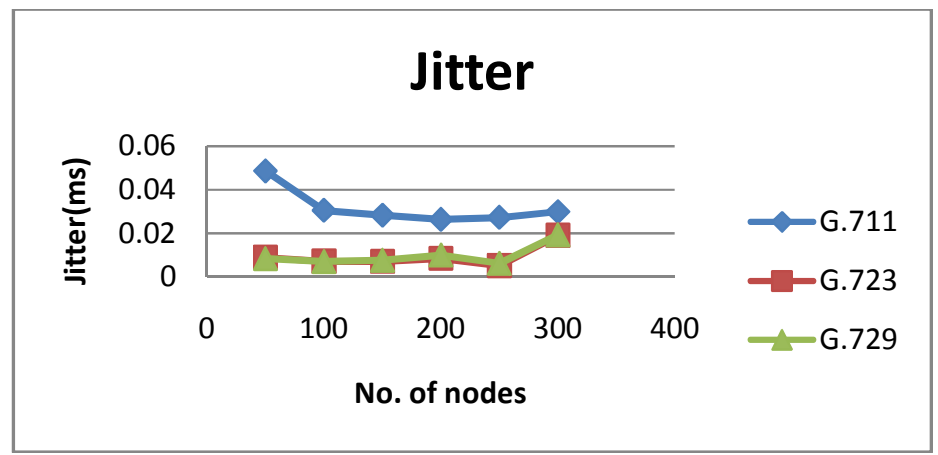

Fig. 13 Jitter vs Number of nodes

From the above figure and below table describes, the variation of the codec is minimum and approximately constant throughout the simulation. The average voice jitter variation in case of codec G.711 is higher than the other two codecs at the earlier time of simulation. But after some time it falls down. The jitter variation in case of G.729 lies between two other audio codecs.

\begin{tabular}{|c|c|c|c|}
\hline \multicolumn{4}{|c|}{ Jitter } \\
\hline No of Nodes & G.711 & $\mathbf{G . 7 2 3}$ & $\mathbf{G . 7 2 9}$ \\
\hline 50 & 0.048555 & 0.008912 & 0.008303 \\
\hline 100 & 0.030457 & 0.007098 & 0.006909 \\
\hline 150 & 0.028252 & 0.006987 & 0.007538 \\
\hline 200 & 0.026274 & 0.008378 & 0.009915 \\
\hline 250 & 0.027239 & 0.005044 & 0.006063 \\
\hline 300 & 0.029873 & 0.018948 & 0.019216 \\
\hline
\end{tabular}

Table 12 Readings of Jitter vs Number of nodes 
The voice jitter threshold for smooth communication in VOIP network is about $1 \mathrm{~ms}$ so audio codec G.729 gives better results than audio codecs G.711 and G.723 respectively.

\subsection{CODEC-RATE ADAPTATION}

\subsubsection{Delay}

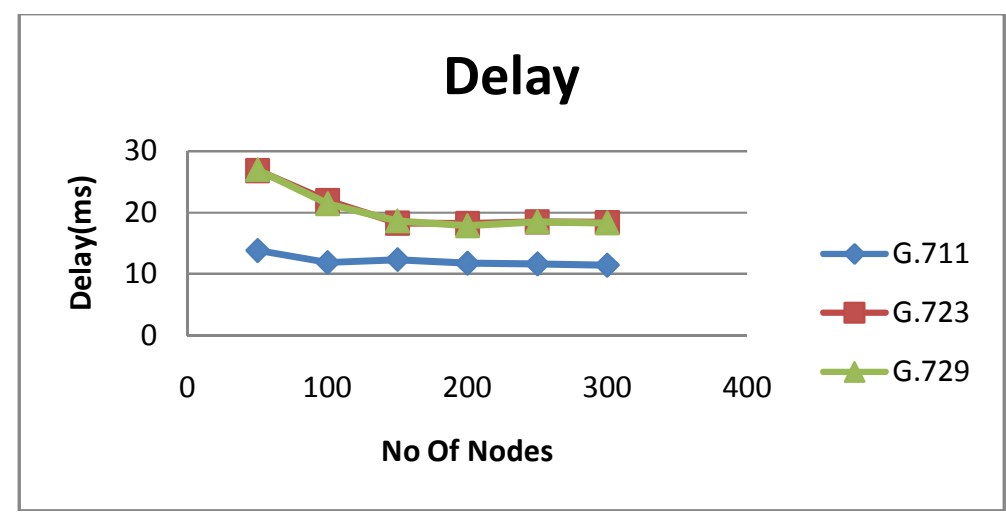

Fig.14 Delay vs Number of nodes

\begin{tabular}{|c|c|c|c|}
\hline \multicolumn{4}{|c|}{ Delay } \\
\hline No of Nodes & G.711 & $\mathbf{G . 7 2 3}$ & $\mathbf{G . 7 2 9}$ \\
\hline 50 & 13.8342 & 26.8252 & 26.988 \\
\hline 100 & 11.8812 & 21.933 & 21.451 \\
\hline 150 & 12.3554 & 18.3049 & 18.6247 \\
\hline 200 & 11.7479 & 18.219 & 17.8569 \\
\hline 250 & 11.6647 & 18.5019 & 18.5019 \\
\hline 300 & 11.449 & 18.3837 & 18.3136 \\
\hline
\end{tabular}

Table 13 Readings of Delay vs Number of nodes

From the above figure and table describes Delay was gained at destination node against various dimension of networks and varied the simulation time uniformly for each codec. This data may be delivered over a physical or logical link, or pass through a certain network node. it is clear that G.729 gives more delay when the nodes are more. G.711 and G.723 codecs gives less delay. G.729 had a high delay. From these graphs it is clear that delay decrease with increase in nonmobility nodes.

\subsubsection{MOS}

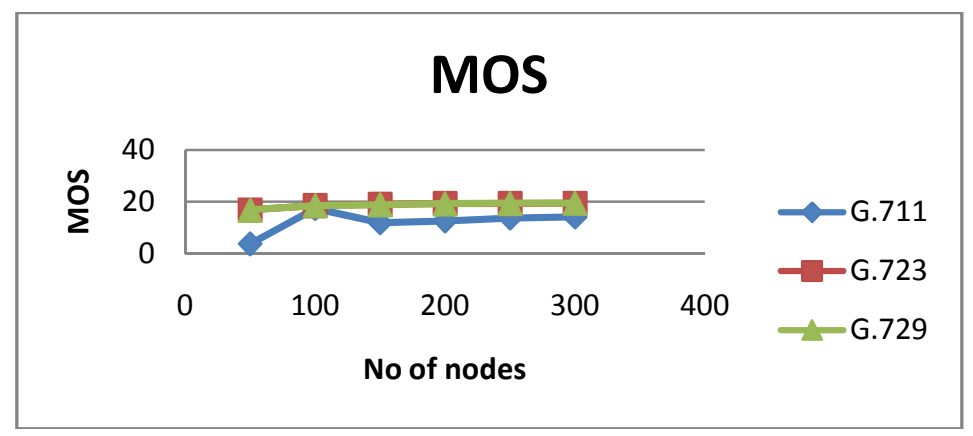

Fig.15. MOS vs Number of nodes 


\begin{tabular}{|c|c|c|c|}
\hline \multicolumn{4}{|c|}{ MOS } \\
\hline No of Nodes & G.711 & G.723 & G.729 \\
\hline 50 & 3.67614 & 16.7733 & 16.7452 \\
\hline 100 & 17.2539 & 18.3478 & 18.384 \\
\hline 150 & 11.8256 & 18.8024 & 18.7271 \\
\hline 200 & 12.5001 & 19.1248 & 19.1387 \\
\hline 250 & 13.6271 & 19.2368 & 19.2439 \\
\hline 300 & 14.012 & 19.3194 & 19.3139 \\
\hline
\end{tabular}

Table 14 Readings of MOS vs Number of nodes

The most widely used QoS metric in VOIP applications is MOS. The MOS value describes the voice perception quality. The average MOS value for the three codecs is represented in figure above. From the graph it is observed that MOS value increases as the nodes increases. Codecs G.711 and G.723 have acceptable MOS value G.723 and G.729, respectively. On the other hand, the MOS value for G.729 is 19.3738 which indicate that the quality of service is good if this codec is used.

\subsubsection{PDR}

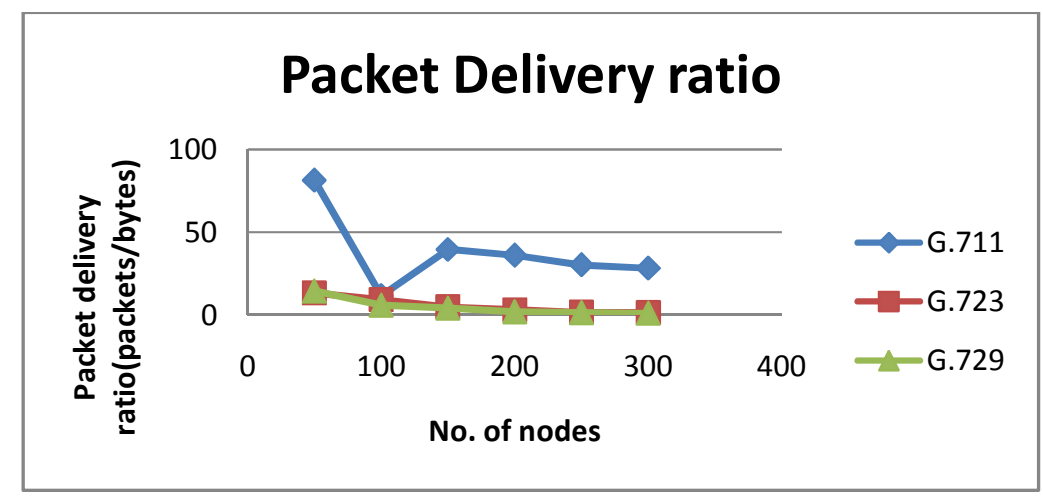

Fig.16 PDR vs Number of nodes

\begin{tabular}{|c|c|c|c|}
\hline \multicolumn{4}{|c|}{ Packet Delivery ratio } \\
\hline No of Nodes & G.711 & G.723 & G.729 \\
\hline 50 & 81.3187 & 13.2897 & 14.4356 \\
\hline 100 & 11.6667 & 9.26613 & 5.97557 \\
\hline 150 & 39.5095 & 4.89773 & 4.18666 \\
\hline 200 & 36.044 & 3.15673 & 2.0679 \\
\hline 250 & 30.2632 & 1.65714 & 1.53505 \\
\hline 300 & 28.2869 & 1.54652 & 1.17401 \\
\hline
\end{tabular}

Table 15 Readings of PDR vs Number of nodes

From the above figure and table describes the average voice PDR comparison using different codecs. From the figure, the variation of the codec is minimum after 100 nodes and approximately varying throughout the simulation. The average voice throughput variation in case of codec G.723 is lower than the other two codecs at the earlier time of simulation. The PDR variation in case of G.729 lies between two other audio codecs. So audio codec G.723 gives better results than audio codecs G.711 and G.729 respectively. 


\subsubsection{Throughput}

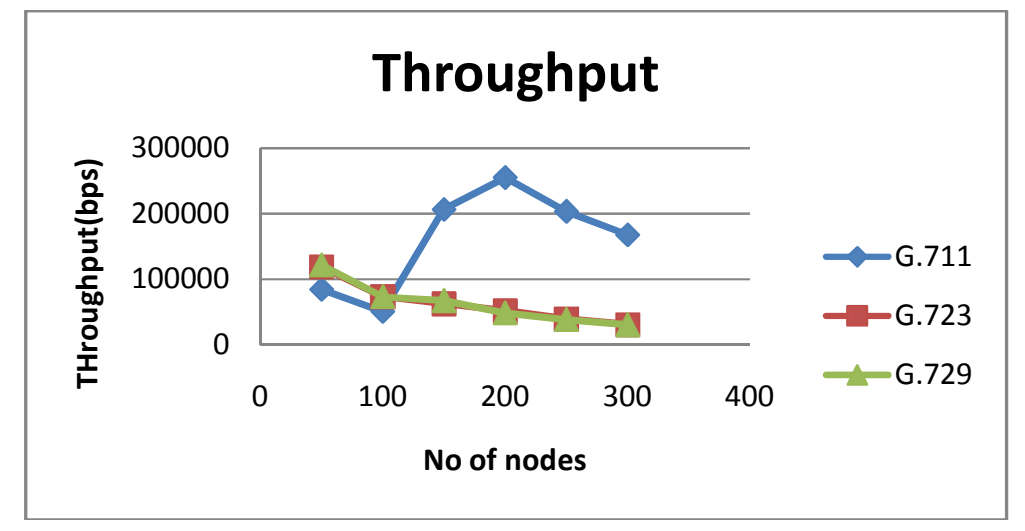

Fig.17. Throughput vs Number of nodes

\begin{tabular}{|c|c|c|c|}
\hline \multicolumn{5}{|c|}{ Throughput } \\
\hline No of Nodes & G.711 & G.723 & G.729 \\
\hline 50 & 83981.4 & 118617 & 121296 \\
\hline 100 & 50447.9 & 73229.2 & 73132.1 \\
\hline 150 & 206104 & 62387.5 & 67257.9 \\
\hline 200 & 254762 & 51735.5 & 48410.9 \\
\hline 250 & 203085 & 39101.3 & 38431.8 \\
\hline 300 & 167279 & 30617.7 & 30371.3 \\
\hline
\end{tabular}

Table 16. Readings of Throughput vs Number of nodes

From the above figure and table describes the average voice throughput comparison using different codecs. From the figure, the variation of the codec is minimum and approximately varying throughout the simulation. The average voice throughput variation in case of codec G.711 is higher than the other two codecs at the earlier time of simulation. But after some time it falls down. The throughput variation in case of G.723 lies between two other audio codecs. So audio codec G.729 gives better results than audio codecs G.711 and G.723 respectively.

\subsubsection{Jitter}

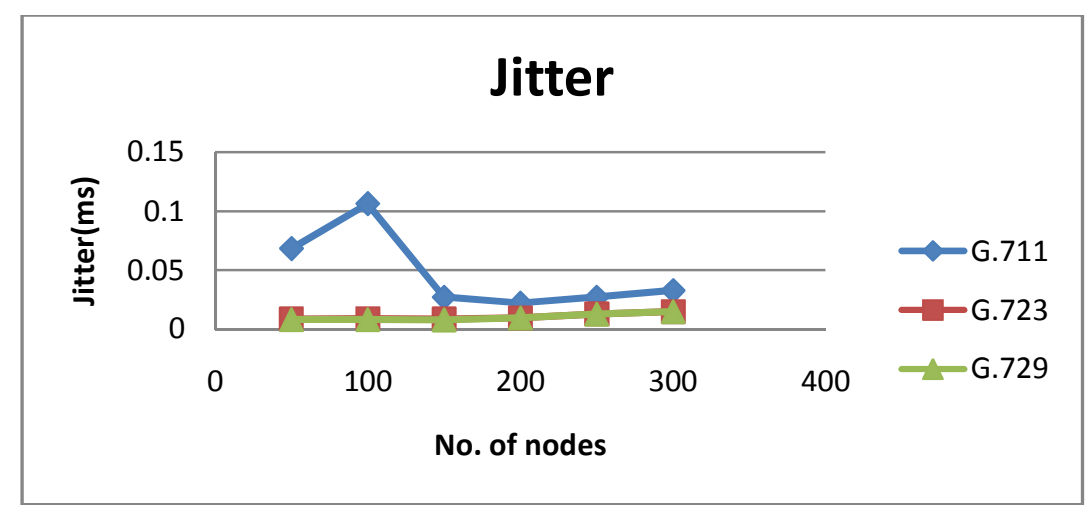

Fig.18 Jitter vs Number of nodes 


\begin{tabular}{|c|c|c|c|}
\hline \multicolumn{4}{|c|}{ Jitter } \\
\hline No of Nodes & G.711 & $\mathbf{G . 7 2 3}$ & G.729 \\
\hline 50 & 0.068239 & 0.008498 & 0.008088 \\
\hline 100 & 0.106221 & 0.008641 & 0.008145 \\
\hline 150 & 0.027136 & 0.008401 & 0.00785 \\
\hline 200 & 0.021992 & 0.009492 & 0.009489 \\
\hline 250 & 0.0271 & 0.012794 & 0.012794 \\
\hline 300 & 0.032781 & 0.014816 & 0.014902 \\
\hline
\end{tabular}

Table 17 Readings of Jitter vs Number of nodes

From the above figure and table, the variation of the codec is minimum and approximately constant throughout the simulation. The average voice jitter variation in case of codec G.711 is higher than the other two codecs at the earlier time of simulation. But after some time it falls down. The jitter variation in case of G.729 lies between two other audio codecs. The voice jitter threshold for smooth communication in VOIP network is about 1ms so audio codec G.729 gives better results than audio codecs G.711 and G.723 respectively.

\begin{tabular}{|c|c|c|c|c|c|c|c|c|c|}
\hline \multicolumn{4}{|c|}{ Codec Information } & \multicolumn{6}{|c|}{ Bandwidth Calculations } \\
\hline $\begin{array}{c}\text { Codec \& } \\
\text { Bit Rate } \\
\text { (Kbps) }\end{array}$ & $\begin{array}{c}\text { Code } \\
\text { c } \\
\text { Sam } \\
\text { ple } \\
\text { Size } \\
\text { (Byte } \\
\text { s) }\end{array}$ & $\begin{array}{c}\text { Code } \\
\text { c } \\
\text { Samp } \\
\text { le } \\
\text { Inter } \\
\text { val } \\
\text { (ms) }\end{array}$ & $\begin{array}{l}\text { Mean } \\
\text { Opini } \\
\text { on } \\
\text { Score } \\
\text { (MOS } \\
\text { ) }\end{array}$ & $\begin{array}{c}\text { Voice } \\
\text { Paylo } \\
\text { ad } \\
\text { Size } \\
\text { (Byte } \\
\text { s) }\end{array}$ & $\begin{array}{c}\text { Voice } \\
\text { Paylo } \\
\text { ad } \\
\text { Size } \\
(\mathrm{ms})\end{array}$ & $\begin{array}{c}\text { Pack } \\
\text { ets } \\
\text { Per } \\
\text { Secon } \\
\text { d } \\
\text { (PPS) }\end{array}$ & $\begin{array}{l}\text { Bandwi } \\
\text { dth MP } \\
\text { or } \\
\text { FRF.12 } \\
\text { (Kbps) }\end{array}$ & $\begin{array}{c}\text { Bandwi } \\
\text { dth } \\
\text { w/cRTP } \\
\text { MP or } \\
\text { FRF.12 } \\
\text { (Kbps) }\end{array}$ & $\begin{array}{l}\text { Bandwi } \\
\text { dth } \\
\text { Etherne } \\
\text { t (Kbps) }\end{array}$ \\
\hline $\begin{array}{l}\text { G.711 (64 } \\
\text { Kbps) }\end{array}$ & $\begin{array}{c}80 \\
\text { Bytes }\end{array}$ & $10 \mathrm{~ms}$ & 4.1 & $\begin{array}{c}160 \\
\text { Bytes }\end{array}$ & $20 \mathrm{~ms}$ & 50 & $\begin{array}{l}82.8 \\
\mathrm{Kbps}\end{array}$ & $\begin{array}{l}67.6 \\
\text { Kbps }\end{array}$ & $\begin{array}{c}87.2 \\
\text { Kbps }\end{array}$ \\
\hline $\begin{array}{l}\text { G.729 (8 } \\
\text { Kbps) }\end{array}$ & $\begin{array}{c}10 \\
\text { Bytes }\end{array}$ & $10 \mathrm{~ms}$ & 3.92 & $\begin{array}{c}20 \\
\text { Bytes }\end{array}$ & $20 \mathrm{~ms}$ & 50 & $\begin{array}{l}26.8 \\
\text { Kbps }\end{array}$ & $\begin{array}{l}11.6 \\
\text { Kbps }\end{array}$ & $\begin{array}{l}31.2 \\
\text { Kbps }\end{array}$ \\
\hline $\begin{array}{l}\text { G.723.1 } \\
(6.3 \mathrm{Kbps})\end{array}$ & $\begin{array}{c}24 \\
\text { Bytes }\end{array}$ & $30 \mathrm{~ms}$ & 3.9 & $\begin{array}{c}24 \\
\text { Bytes }\end{array}$ & $30 \mathrm{~ms}$ & 33.3 & $\begin{array}{l}18.9 \\
\text { Kbps }\end{array}$ & $8.8 \mathrm{Kbps}$ & $\begin{array}{l}21.9 \\
\text { Kbps }\end{array}$ \\
\hline $\begin{array}{l}\text { G. } 723.1 \\
(5.3 \mathrm{Kbps})\end{array}$ & $\begin{array}{c}20 \\
\text { Bytes }\end{array}$ & $30 \mathrm{~ms}$ & 3.8 & $\begin{array}{c}20 \\
\text { Bytes }\end{array}$ & $30 \mathrm{~ms}$ & 33.3 & $\begin{array}{c}17.9 \\
\text { Kbps }\end{array}$ & $7.7 \mathrm{Kbps}$ & $\begin{array}{l}20.8 \\
\text { Kbps }\end{array}$ \\
\hline $\begin{array}{l}\text { G.726(32 } \\
\text { Kbps) }\end{array}$ & $\begin{array}{c}20 \\
\text { Bytes }\end{array}$ & $5 \mathrm{~ms}$ & 3.85 & $\begin{array}{c}80 \\
\text { Bytes }\end{array}$ & $20 \mathrm{~ms}$ & 50 & $\begin{array}{l}50.8 \\
\text { Kbps }\end{array}$ & $\begin{array}{l}35.6 \\
\text { Kbps }\end{array}$ & $\begin{array}{l}55.2 \\
\text { Kbps }\end{array}$ \\
\hline $\begin{array}{l}\text { G.726 (24 } \\
\text { Kbps) }\end{array}$ & $\begin{array}{c}15 \\
\text { Bytes }\end{array}$ & $5 \mathrm{~ms}$ & 3.85 & $\begin{array}{c}70 \\
\text { Bytes }\end{array}$ & $20 \mathrm{~ms}$ & 50 & $\begin{array}{l}42.8 \\
\text { Kbps }\end{array}$ & $\begin{array}{l}27.6 \\
\text { Kbps }\end{array}$ & $\begin{array}{l}47.2 \\
\text { Kbps }\end{array}$ \\
\hline $\begin{array}{l}\text { G.728 (16 } \\
\text { Kbps) }\end{array}$ & $\begin{array}{c}10 \\
\text { Bytes }\end{array}$ & $5 \mathrm{~ms}$ & 3.61 & $\begin{array}{c}60 \\
\text { Bytes }\end{array}$ & $30 \mathrm{~ms}$ & 33.3 & $\begin{array}{l}28.5 \\
\text { Kbps }\end{array}$ & $\begin{array}{c}18.4 \\
\text { Kbps }\end{array}$ & $\begin{array}{l}31.5 \\
\text { Kbps }\end{array}$ \\
\hline $\begin{array}{l}\text { G722 } 64 \mathrm{k} \\
(64 \mathrm{~Kb} p)\end{array}$ & $\begin{array}{c}80 \\
\text { Bytes }\end{array}$ & $10 \mathrm{~ms}$ & 4.13 & $\begin{array}{c}160 \\
\text { Bytes }\end{array}$ & $20 \mathrm{~ms}$ & 50 & $\begin{array}{l}82.8 \\
\text { Kbps }\end{array}$ & $\begin{array}{l}67.6 \\
\text { Kbps }\end{array}$ & $\begin{array}{l}87.2 \\
\text { Kbps }\end{array}$ \\
\hline $\begin{array}{l}\text { ilbc_mode } \\
20 \\
(15.2 \mathrm{Kbps} \\
\end{array}$ & $\begin{array}{c}38 \\
\text { Bytes }\end{array}$ & $20 \mathrm{~ms}$ & NA & $\begin{array}{c}38 \\
\text { Bytes }\end{array}$ & $20 \mathrm{~ms}$ & 50 & $\begin{array}{l}34.0 \\
\text { Kbps }\end{array}$ & $\begin{array}{c}18.8 \\
\text { Kbps }\end{array}$ & $\begin{array}{l}38.4 \\
\text { Kbps }\end{array}$ \\
\hline $\begin{array}{l}\text { ilbc_mode } \\
30 \\
(13.33 \mathrm{Kbp} \\
\text { s) }\end{array}$ & $\begin{array}{c}50 \\
\text { Bytes }\end{array}$ & $30 \mathrm{~ms}$ & NA & $\begin{array}{c}50 \\
\text { Bytes }\end{array}$ & $30 \mathrm{~ms}$ & 33.3 & $\begin{array}{c}25.867 \\
\text { Kbps }\end{array}$ & $\begin{array}{l}15.73 \\
\text { Kbps }\end{array}$ & $\begin{array}{l}28.8 \\
\text { Kbps }\end{array}$ \\
\hline
\end{tabular}

Table18. Comparison of different Codecs. 


\section{CONCLUSION}

In this project, So far the comparison between codec adaptive, rate adaptive, codec and rate adaptive and non-adaptive methods have been done and results show that in most of the cases when the congestion is low or moderate, rate adaptation improves the perceived quality and results in lower load and lower end-to end delay and lower packet loss compared to non-adaptive methods. However, in terms of severe congestion, codec adaptation act better than rate adaptation since it has a big effect on the speech output rate. Accordingly, since rate adaptation has less impact on the system in terms of more simplicity and lower coding cost, the algorithm will be started by rate adaptation in the case of low congestion. If the system becomes highly congested where rate adaptation is not enough, the algorithm will commence codec adaptation. Furthermore, in the critical situation where even codec adaptation could not rectify the congestion, both approaches can work together.

\section{REFERENCES:}

[1] Performance Analysis of VoIP Codecs over WiMAX, Khaled Alutaibi

[2] H. Kazemitabar, A. Md. Said UniversitiyTechnology PETRONAS hanieh80@gmail.com Adaptive QoS Control Approaches for VoIP Over Multi-Rate WLAN's.

[3] A survey of quality of service in IEEE802.11 Networks,HUA ZHU, MING LI, IMRICH CHLAMTAC, AND B. PRABHAKARANTHE UNIVERSITY OF TEXAS AT DALLAS.

[4] Priority Based Dynamic Rate Control for VoIP Traffic, Fariza Sabrina CSIRO ICT Centre, Sydney, Australia.

[5] VoIP codec adaptation algorithm in multirate 802.11 WLANs, Anna Sfairopoulou, Carlos Macilan, Boris Bellalta.

[6] A new method for VoIP Quality of Service control using combined adaptive sender rate and priority marking, Zizhi Qiao, Lingfen Sun, Nicolai Heilemann and Emmanuel Ifeachor.

[7] VoIP over Wireless LAN Survey, Răzvan Beuran,Internet Research Center Japan Advanced Institute of Science and Technology (JAIST).

[8] ITU-T

[9] Adaptive QoS Control Approaches for VoIP Over Multi-Rate WLANs, H. Kazemitabar, A. Md. Said Universiti Teknologi PETRONAS Bandar Seri Iskandar, 31750 Tronoh, Perak, Malaysia.

[10] Performance Analysis Of VOIP Codec's With QoS, Parameters, Priyanka Grover1, Meenakshi Chawla2 Dept. of Computer Engineering, TIT\&S, Bhiwani.

[11] PERFORMANCE ANALYSIS OF VOIP TRAFFIC OVER INTEGRATING WIRELESS LAN AND WAN USING DIFFERENT CODECS, Ali M. Alsahlany, Department of Communication Engineering, Al-Najaf Technical College, Foundation of Technical Education, Iraq.

[12] Performance Analysis of various Codecs Schemes of VOIP over WiMAX, M-Tech Student\#1 ,Assit. Prof. *2 \& Dept. of CSE., Manav Rachna International University, Faridabad, Haryana, India.

[13] Performance Analysis of VOIP Codes under Different Mobility Pattern,Manpreet Kaur.

[14] Performance Evaluation of The Quality of VoIP Over WLAN Codecs, H.A. Ifijeh, F.E. Idachaba, and I.B. Oluwafemi

[15] R. M. Hirannaiah, et al., "Influence of Codecs on Adaptive Jitter Buffer Algorithm," in Vehicular Technology Conference, 2007.VTC-2007 Fall. 2007 IEEE 66th, 2007, pp. 2015-2019. 
[16] M. Narbutt and L. Murphy. (2003, VoIP Playout Buffer Adjustment using Adaptive Estimation of Network Delays. Research Publications.

[17] S. Madhani, et al., "Optimized Adaptive Jitter Buffer Design for Wireless Internet Telephony," in Global Telecommunications Conference, 2007. GLOBECOM '07. IEEE, 2007, pp. 52485253.

[18] L. Jing and N. Zhisheng, "An adaptive receiver buffer adjust algorithm for VoIP applications considering voice characters," in Communications, 2004 and the 5th International Symposiumon Multi-Dimensional Mobile Communications Proceedings. The 2004 Joint Conference of the 10th Asia-Pacific Conference on, 2004, pp. 597-601 vol.2.

[19] M. Baratvand, et al., "Jitter-Buffer Management for VoIP over Wireless LAN in a Limited Resource Device," in Networkingand Services, 2008. ICNS 2008. Fourth InternationalConference on, 2008, pp. 90-95.

[20] J. Light and A. Bhuvaneshwari, "Performance analysis of audio codecs over real-time transmission protocol (RTP) for voice services over Internet protocol," in Communication Networksand Services Research, 2004. Proceedings. Second AnnualConference on, 2004, pp. 351-356.

[21] O. Hersent, et al., Beyond VoIP Protocols: Understanding Voice Technology and Networking Techniques for IP Telephony:Wiley, 2005.

[22] B. Ngamwongwattana, "Sync \& Sense Enabled Adaptive Packetization VoIP," Phd Pittsburgh, USA, 2007.

[23] S. Garg and M. Kappes, "Can I add a VoIP call?," in Communications, 2003. ICC '03. IEEE InternationalConference on, 2003, pp. 779-783 vol.2. 